\title{
Airway pressure release ventilation versus conventional ventilation for the management of pediatric acute respiratory distress syndrome: do we have an answer?
}

\author{
Krishna Kishore Umapathi, Maroun J. Mhanna \\ Division of Pediatric Critical Care, Department of Pediatrics, Case Western Reserve University School of Medicine, Metro Health Medical Center, \\ Cleveland, OH, USA \\ Correspondence to: Maroun J. Mhanna, MD, MPH. Department of Pediatrics, 2500, Metro Health Drive, Cleveland, OH 44129, USA. \\ Email: mmhanna@metrohealth.org. \\ Provenance: This is an invited Editorial commissioned by the Section Editor Zhiheng Xu (State Key Laboratory of Respiratory Disease, Guangzhou \\ Institute of Respiratory Disease, Department of Intensive Care, The First Affiliated Hospital of Guangzhou Medical University, Guangzhou, China). \\ Comment on: Lalgudi Ganesan S, Jayashree M, Singhi SC, et al. Airway Pressure Release Ventilation in Pediatric Acute Respiratory Distress \\ Syndrome: a Randomized Controlled Trial. Am J Respir Crit Care Med 2018;198:1199-7.
}

Submitted Sep 17, 2018. Accepted for publication Oct 01, 2018.

doi: $10.21037 /$ jtd.2018.10.23

View this article at: http://dx.doi.org/10.21037/jtd.2018.10.23

Acute respiratory distress syndrome (ARDS) is a significant cause of respiratory failure in children who need to be admitted to the pediatric intensive care unit (PICU). The American-European Consensus Conference (AECC) and the Berlin definitions of ARDS did not address the pediatric specific practices and comorbidities $(1,2)$. Therefore, the Pediatric Acute Lung Injury Consensus Conference (PALICC) bridged the gap to define pediatric ARDS (PARDS). The definition of PARDS differed from the adult's definition by using Oxygenation Index (OI) to replace $\mathrm{PaO}_{2} / \mathrm{FiO}_{2}$ ratio, Oxygen Saturation Index to measure the severity of illness, and deemphasized the importance of the radiographic criteria (3).

The management of PARDS has mainly focused on the diagnosis and treatment of the underlying cause and on the attempt to prevent secondary lung injury. Multiple ventilator strategies have been used by pediatric critical care specialists to improve lung recruitment, optimize positive end expiratory pressure (PEEP), and ventilate at a lower tidal volume (TV). Low TV conventional ventilation, high frequency oscillatory ventilation (HFOV) and airway pressure release ventilation (APRV) have been used, but there is a paucity of pediatric literature to show superiority of one mode of ventilation over another. In a prospective, randomized controlled trial, recently published in the American fournal of Respiratory and Critical Care Medicine (4), Lalgudi Ganesan et al. compared
APRV to low TV conventional ventilation in children with PARDS. After enrollment of 52 patients, the study was terminated following an interim analysis showing an increased mortality in patients treated with APRV. Fifty three percent of children who were treated with APRV died vs. twenty seven percent of the children who were treated with conventional ventilation.

The authors must be applauded for their efforts to study such an important subject in a prospective randomized controlled manner. However, in reviewing the study, there were striking differences at baseline between the studied groups. APRV patients were younger and had a higher percentage of primary ARDS than their controls. There were statistically significant differences in the severity of ARDS between the two groups. Children who were ventilated with APRV had a higher $\mathrm{PaO}_{2} / \mathrm{FiO}_{2}$ ratio, and a higher OI than their controls, denoting that the APRV group was at a disadvantage at enrollment. There was no difference in the primary outcome of ventilator free days between the two groups. However, there was a difference in 28-day mortality in patients treated with APRV $(R R=3.2)$, although non-statically significant $(\mathrm{P}>0.05)$ but clinically relevant. Even after adjusting for the severity of ARDS, mortality was higher in the APRV group ( $R R=2.02)$, which led to the termination of the study.

The authors concluded that there is a trend towards a 
higher mortality with APRV. Although this is a significant statement with widespread practice implication, the findings should be interpreted cautiously as there are multiple limitations to the study. It is a single center study, which was underpowered by early termination and unstratified randomization. A future multicenter, randomized controlled, and appropriately stratified trial is needed to determine if APRV is a viable option in children with ARDS. Stratification is paramount for any future studies, to address the potential confounders of Ganesan's study. At baseline, children who were ventilated with APRV were relatively younger, and had a higher severity of ARDS than their controls confounders that would affect morbidity and mortality.

In Ganesan's study, children who were ventilated with APRV had a higher mean airway pressure (MAP) and they were more often spontaneously breathing. Younger children have a greater chest wall compliance than older children. Therefore, for the same pressures, volumes may be significantly higher. And if patients on APRV have increased spontaneous breathing with potentially higher generated TVs, spontaneous breathing might become theoretically detrimental, especially in younger children (those who were randomized to APRV).

Although higher MAPs in APRV might affect the hemodynamic stability by decreasing the cardiac output and renal perfusion $(5,6)$, it has been shown that in children with ARDS, despite a higher MAP, APRV does not affect blood pressure or urine output (7) and can even restore hemodynamic stability faster than conventional ventilation (8).

The advantage of using APRV is to maximize recruitment of alveoli and allow spontaneous breathing thereby minimizing barotrauma. In animal models, APRV has been shown to successfully prevent the development of ARDS (9). In adults, a meta-analysis of six randomized controlled studies showed no significant differences in morbidity or mortality between patients treated with APRV or conventional ventilation (10). The role of APRV in pediatric critically ill patients has always been a debate. Although there have been multiple case reports and case series of APRV use in PARDS, Lalgudi Ganesan et al. report the first randomized controlled trial comparing APRV to conventional ventilation in PARDS.

In conclusion, the study by Lalgudi Ganesan and colleagues is very important. It is the first, randomized controlled trial comparing APRV to conventional ventilation. Unfortunately, the differences between the group of patients who were enrolled, and the lack of stratification at randomization have prevented reaching a conclusion and a definitive answer.

\section{Acknowledgements}

None.

\section{Footnote}

Conflicts of Interest: The authors have no conflicts of interest to declare.

\section{References}

1. Bernard GR, Artigas A, Brigham KL, et al. The American-European Consensus Conference on ARDS. Definitions, mechanisms, relevant outcomes, and clinical trial coordination. Am J Respir Crit Care Med 1994;149:818-24.

2. ARDS Definition Task Force, Ranieri VM, Rubenfeld GD, et al. Acute respiratory distress syndrome: the Berlin Definition. JAMA 2012;307:2526-33.

3. Khemani RG, Smith LS, Zimmerman JJ, et al. Pediatric acute respiratory distress syndrome: definition, incidence, and epidemiology: proceedings from the Pediatric Acute Lung Injury Consensus Conference. Pediatr Crit Care Med 2015;16:S23-40.

4. Lalgudi Ganesan S, Jayashree M, Singhi SC, et al. Airway Pressure Release Ventilation in Pediatric Acute Respiratory Distress Syndrome: a Randomized Controlled Trial. Am J Respir Crit Care Med 2018;198:1199-7.

5. Long Y, Su L, Zhang Q, et al. Elevated Mean Airway Pressure and Central Venous Pressure in the First Day of Mechanical Ventilation Indicated Poor Outcome. Crit Care Med 2017;45:e485-92.

6. Panico FF, Troster EJ, Oliveira CS, et al. Risk Factors for Mortality and Outcomes in Pediatric Acute Lung Injury/ Acute Respiratory Distress Syndrome. Pediatr Crit Care Med 2015;16:e194-200.

7. Kamath SS, Super DM, Mhanna MJ. Effects of airway pressure release ventilation on blood pressure and urine output in children. Pediatr Pulmonol 2010;45:48-54.

8. Hussain A, Lababidi H, Mir A, et al. The effectiveness of hemodynamic status among septic shock patients by airway pressure release ventilation (APRV) in comparison with conventional mode ventilation $(\mathrm{CMV})$ : a retrospective cohort study. Am J Respir Crit Care Med 
2016;193:A7040.

9. Roy S, Habashi N, Sadowitz B, et al. Early airway pressure release ventilation prevents ARDS-a novel preventive approach to lung injury. Shock 2013;39:28-38.

Cite this article as: Umapathi KK, Mhanna MJ. Airway pressure release ventilation versus conventional ventilation for the management of pediatric acute respiratory distress syndrome: do we have an answer? J Thorac Dis 2018;10(Suppl 33):S4085-S4087. doi: 10.21037/jtd.2018.10.23
10. Bajaj A, Rathor P, Kabak B, et al. Efficacy of Airway Pressure Release Ventilation in critically ill patients: a meta-analysis of randomized controlled trials. Am J. Respir Crit Care Med 2015;191:A1195. 